\title{
Frustration-induced magic number clusters of colloidal magnetic particles
}

\author{
Larysa Baraban, ${ }^{1, *}$ Denys Makarov, ${ }^{1}$ Manfred Albrecht, ${ }^{1,2}$ Nicolas Rivier, ${ }^{3}$ Paul Leiderer, ${ }^{1}$ and Artur Erbe ${ }^{1}$ \\ ${ }^{1}$ Department of Physics, University of Konstanz, Universitätstrasse 10, Konstanz, D-78457, Germany \\ ${ }^{2}$ Surface and Interface Physics, Chemnitz University of Technology, D-09107 Chemnitz, Germany \\ ${ }^{3}$ IPCMS (Institut de Physique et Chimie des Materiaux de Strasbourg), 23 rue du Loess, Boîte Postale 43, 67034, \\ Strasbourg Cedex 2, France
}

(Received 19 November 2007; published 21 March 2008)

\begin{abstract}
We report the formation of stable two-dimensional clusters consisting of long-range-interacting colloidal particles with predefined magnetic moments. The symmetry and arrangement of the particles within the cluster are imposed by the magnetic frustration. By satisfying the criteria of stability, a series of magic number clusters is formed. The magic clusters are close packed and have compensating magnetic moments and chirality. Thus, the system can be regarded as a classical mesoscopic model for spin arrangements in two-dimensional triangular antiferromagnets, although the exact nature of the interactions between the macroscopic magnetic moments is different.
\end{abstract}

PACS number(s): 82.70.Dd, 41.20.Gz, 75.50.Ee

\section{INTRODUCTION}

Systems of interacting colloidal particles have proven to be excellent model systems for many processes on the microscopic scale [1-4]. Up to now most experiments on colloids were performed using hard spheres [1,5], particles relying on properties provided by electrical charges [6], or particles with magnetic moments, which depend on the application of an external magnetic field [4]. A variety of studies was dedicated to obtain different types of crystal stoichiometries by tuning the interaction potential between the colloidal particles in an ensemble [2,7]. In this way, the modeling of the properties of the ionic crystal was demonstrated and the possible stabilization of new unknown phases was predicted [8].

There is a great diversity of phenomena governed by ordering of magnetic moments in crystals [9-14], which also need a convenient macroscopic model. For example, the magnetic arrangements have an essential influence on the physical properties of stacked triangular antiferromagnets $[14,15]$ or high- $T_{C}$ superconductors [16], where the interaction between spins defines the nature of the condensed phase. In order to study these phenomena with a colloidal model system, particles with fixed magnetic moments are required. The magnetic properties of many solid phases are determined by the nearest neighbor (NN) interactions of the electron's spins pinned to the lattice geometry, which is determined by the positions of the nuclei. In many such systems a long-range-ordered phase can exist based on these interactions; for example, ferromagnetic ordering due to exchange interactions or antiferromagnetic phases due to dipolar interactions. Other lattice geometries do not allow the formation of such a long-range-ordered phase based on NN interactions. The most basic example of such a system is given

\footnotetext{
*Author to whom correspondence should be addressed. larysa.baraban@uni-konstanz.de
}

by spins on a triangular lattice, where every plaquette is geometrically frustrated. In this configuration the system "looks for" compromise solutions to minimize the total energy, and long-range interactions become important. Nowadays the arrangement of the magnetic moments in crystals is mainly studied by methods that are related to reciprocal space, e.g., neutron scattering $[17,18]$, or inferred from spectroscopic Mössbauer measurements [19,20] or macroscopic quantities like the heat capacity [21]. Theoretical modeling is usually done based on simplifying assumptions, for example restricting free parameters to the directions of the spins. An appropriate macroscopic system for the modeling of the magnetic interaction would help to better understand such complex fundamental phenomena as antiferromagnetic ordering, magnetic spin frustration [10-12], etc. In this regard, magnetic colloidal particles combine many advantages to make them an ideal model system for these purposes. The macroscopic size of the colloids and the ability to tune their interactions easily give the possibility to directly observe the processes of magnetic crystal nucleation and growth, and thus visualize the magnetic interaction within the crystal.

To our knowledge, this work is the first attempt to form a colloidal crystal stabilized only by the magnetic interaction between particles. We used colloids with predefined magnetic moments, which can freely orient in space and therefore they can be thought of as models for particles with a spin. By means of video microscopy, the organization of the magnetic particles in a series of purely two-dimensional magic number clusters $[22,23]$ with certain numbers of particles was visualized. The magnetic moments associated with the particles in the stable cluster were found to lie strictly in plane. The ground state configuration of "magic number" clusters is achieved following the rules of compensation of the total magnetic moment and satisfying the basic principles of the local symmetry. The typical interaction energies between particles are on the same order as the thermal energy $k_{B} T$, which allows the particles to explore the phase space by thermally activated motion until the stable configuration is found. 

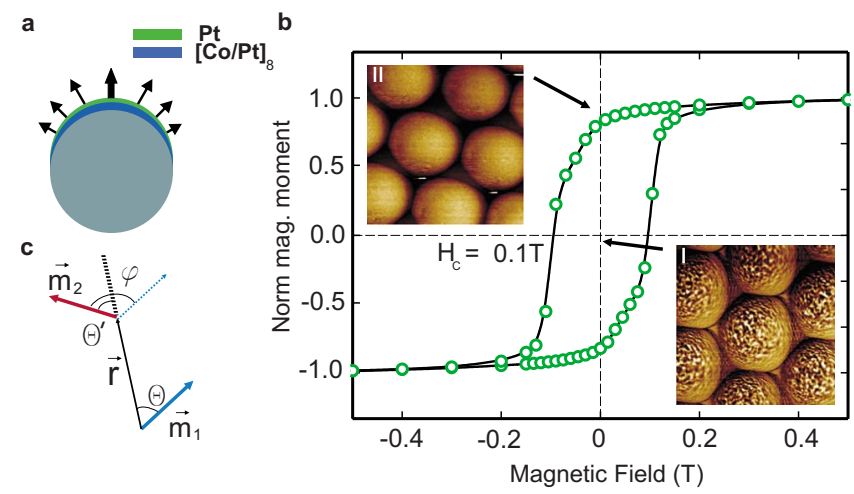

FIG. 1. (Color online) Magnetic moment distribution and magnetic characterization of a particle array covered with a $\mathrm{Co} / \mathrm{Pt}$ multilayer. (a) Schematic sketch of the spatial distribution of the magnetic moments forming a "macrospin." The arrows show the direction of the axis of magnetic anisotropy, which remains perpendicular to the surface of the $\mathrm{Co} / \mathrm{Pt}$ film following the curvature of particles. The spherical shape of the colloids leads to a reduction in Co and Pt thickness along the surface. (b) Hysteresis loop, measured by superconducting quantum interference device (SQUID) magnetometry. Coercive field is equal to $0.1 \mathrm{~T}$. Insets: (I) Magnetic Force Microscopy (MFM) image of magnetic caps with a diameter of $4.75 \mu \mathrm{m}$ in the demagnetized state, revealing a multidomain behavior; (II) MFM image of the caps in the remanent state displays single-domain behavior. (c) Sketch of the interacting magnetic dipoles.

\section{FORMATION OF COLLOIDAL MAGNETIC CLUSTERS}

\section{A. Details of experiment}

In order to serve as a valid model system, the particles have to exhibit monodisperse magnetic properties. The magnetic properties of the colloids are provided by evaporation of $\mathrm{Co} / \mathrm{Pt}$ multilayer films with out-of-plane magnetic anisotropy on top of a densely packed monolayer of silica particles [24,25] with a diameter $d=4.75 \mu \mathrm{m}$, as reported in [26]. In our experiments we use particles with natural silanol (Si-OH) surface groups, which negatively charge the spheres and prevent their undesirable aggregation.

Figure 1 shows a summary of the magnetic properties of the capped colloids. The multilayer of $\mathrm{Co} / \mathrm{Pt}$ exhibits a magnetic anisotropy pointing perpendicular to the surface of the film [27], following the spherical shape of the particle as illustrated in Fig. 1(a). Due to the curved surface of the particle, the center of mass of the magnetic cap is shifted from the geometrical center of the sphere. The hysteresis loop for such a particle array, measured in the out-of-plane field geometry, is presented in Fig. 1(b). In the remanent state the nonzero magnetic moment, directed along the symmetry axis of the cap, is associated with a single-domain particle [28]. This macroscopic magnetic moment forms the so-called macrospin of the particle. A slight reduction in the remanent magnetization is obtained due to the spatial variation of the easy axis of magnetization across the cap surface [29] [Figs. 1(a) and 1(b)].

Covering of the transparent silica particles by a thin metal film leads to an optical inhomogeneity of the spheres, which can be used for the visualization of the orientations of mag-

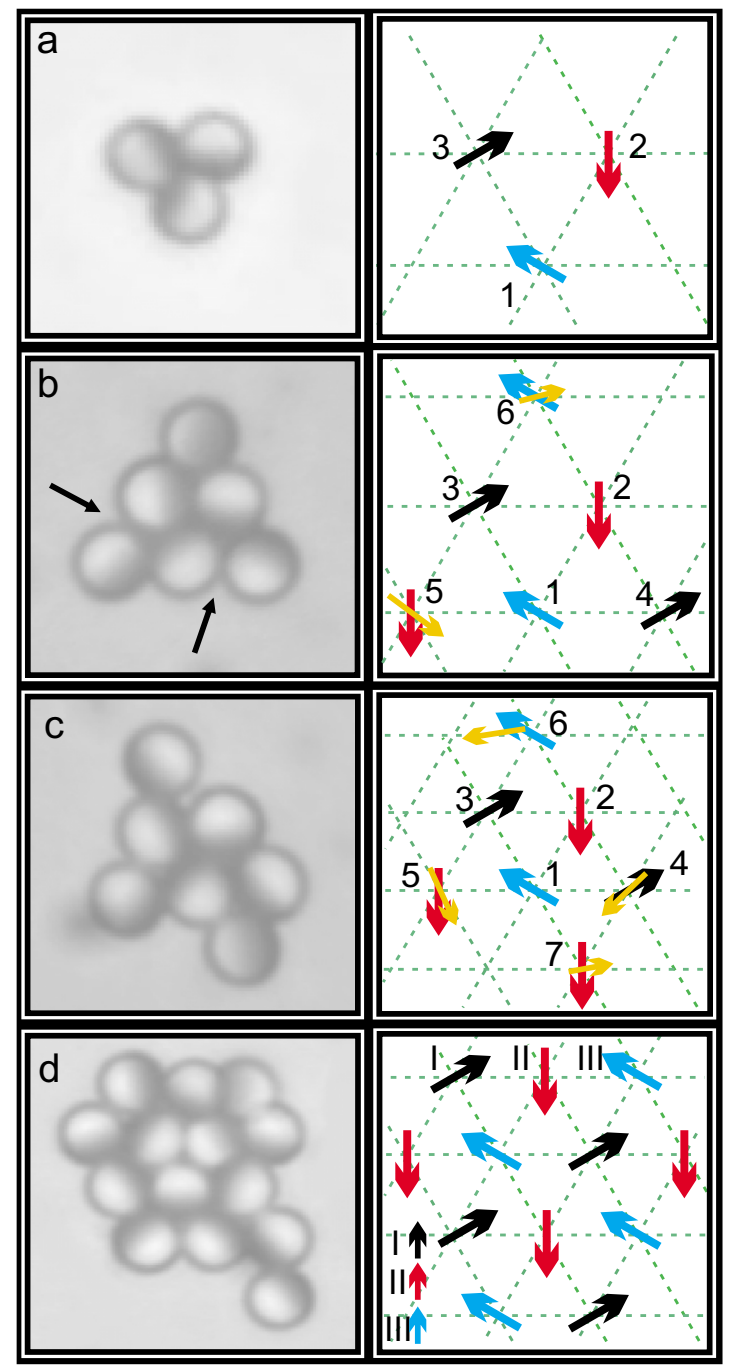

FIG. 2. (Color online) Growth process of magnetic clusters. The pictures on the right display the correspondent schematic view of the ordering of the macrospins on a lattice. Yellow arrows represent the orientations of the magnetic moments that deviate from the preferred directions. (a) The initial nucleus contains three particles. The arrangement of the magnetic moments of particles corresponds to the minimum of energy. (b) depicts the lowest-energy arrangement of six particles. The top particle has its cap tilted slightly out of plane. Black arrows point to the particles with divergent orientation of the macrospins. (c) Visualization of the orientations of the magnetic caps in the case of a seven-particle structure. (d) A structure consisting of 14 particles. 12 particles in the cluster are close packed and all magnetic moments lie strictly in two dimensions. The additional two particles (on the lower right side) do not affect the formed magnetic arrangement.

netic moments in the clusters. The process of magnetic cluster formation was directly imaged by means of video microscopy (Fig. 2). A suspension of capped colloids, prepared by mixing the particles with distilled water, was confined between two glass plates coated by layers of SU-8 (permanent epoxy negative resist), which prevents the particles from getting stuck due to surface charge effects. Optical laser tweezers [30] (wavelength $\lambda=1064 \mathrm{~nm}$ ) were used for manipulating individual particles. The growth of the cluster was 
carried out by gradually adding particles from a reservoir one by one to an initial nucleus.

The colloidal particles experience repulsive hard core interactions and the dipole-dipole interactions of their magnetic moments [Fig. 1(c)]. The interaction energy of two magnetic dipoles is given by

$$
\begin{aligned}
E & =\frac{\mu_{0}}{4 \pi} \frac{m_{1} m_{2}}{r_{12}^{3}} \quad\left(\cos \varphi-3 \cos \theta \cos \theta^{\prime}\right) \\
& =-\frac{a}{2}[\cos \varphi+3 \cos (2 \theta-\varphi)]
\end{aligned}
$$

where $\boldsymbol{m}_{1}$ and $\boldsymbol{m}_{2}$ are the magnetic moments $\left(\left|\boldsymbol{m}_{1}\right|=\left|\boldsymbol{m}_{2}\right|\right.$ $=m), r_{12}$ is the distance between the centers of particles, $\varphi$ is the angle between the directions of the magnetic moments $\left(\boldsymbol{m}_{1} \cdot \boldsymbol{m}_{2}=m^{2} \cos \varphi\right), \theta$ and $\theta^{\prime}$ are the angles between $\boldsymbol{m}_{1}$ and $\boldsymbol{r}\left(\boldsymbol{m}_{1} \cdot \boldsymbol{r}=m r \cos \theta\right)$ and $\boldsymbol{m}_{2}$ and $\boldsymbol{r}\left(\boldsymbol{m}_{2} \cdot \boldsymbol{r}=m r \cos \theta^{\prime}\right)$, respectively [Fig. 1(c)], $\boldsymbol{r}$ is the unit vector connecting the particles, and the angles $\varphi$ and $\theta$ are linked via $\varphi=\theta+\theta^{\prime}$. Note that the prefactor $a$ is equal approximately to $3.5 k_{B} T$.

The energy calculations given in this paper describe the system qualitatively for the case when there is no shift of the center of mass of the magnetic cap with respect to the geometrical center of the particle. This shift, which of course exists in real systems, leads to a change of the ground state energy. Nevertheless, the symmetries of all stable configurations of the magnetic moments stay unchanged.

Because the uncoated part of every sphere carries surface charge, colloidal particles can experience specific (asymmetric) electrostatic repulsion. In order to estimate the influence of this interaction on clustering, in some experiments an additional layer of silicon dioxide was sputtered on top of the $\mathrm{Co} / \mathrm{Pt}$ caps, making the surface of the sphere symmetrically charged. However, this did not affect the process of magnetic cluster formation, i.e., it did not cause changes of lattice geometry or strong repulsion between particles. In addition to this, the ion concentration in the sample was not controlled during experiments, resulting in a very short Debye screening length. Therefore, we do not take into account the electrostatic interactions in the considered system, because they do not influence the arrangement of the magnetic moments.

Another type of interaction that might influence cluster formation is the van der Waals interaction, usually resulting in particle adhesion [31]. As long as particles are able to freely rotate with respect to each other in our experiment, and we detect it in our measurements, the van der Waals forces can also be neglected in the current context.

\section{B. Growth of clusters}

Consider the addition of one magnetic particle to a colloidal cluster consisting of $n$ spheres. In our definition a particle becomes a constituent of the cluster when it touches at least two other particles in the group. The new contact can be considered as stable if the total sum of the pair dipolar interactions between the new particle and each of the $n$ colloidal particles is negative; otherwise the contact is not stable, and the new particle is rejected. The magnetic moment of the cluster changes the orientation of the new macrospin in order to find the new equilibrium state of the system. The new particle itself, in turn, has an influence on the macrospins of the cluster.

The procedure for the creation of a magnetic cluster from a small nucleus is presented in Fig. 2. The images on the left of Fig. 2 are photos of the clusters taken during growth. The pictures on the right represent the corresponding schematic view of the ordering of the macrospins pinned to the lattice: The arrows designated I, II, and III (black, red, and blue) represent the directions of magnetic moments predicted by the symmetry of the system (see below), and the yellow arrows indicate the deviations of the magnetic moments from the preferred directions, as observed experimentally. A short yellow arrow indicates that the magnetic moment has an outof-plane component. The initial nucleus consists of three particles and has the shape of an equilateral triangle, as illustrated in Fig. 2(a), on the left. It can be seen that all magnetic moments strictly lie in the plane parallel to the sample surface. All the macrospins [on the right of Fig. 2(a)] form angles $\varphi=2 \pi / 3$ with respect to each other. If the angles in the system are counted counterclockwise, the values of $\theta$ and $\theta^{\prime}$ are given by $\theta_{1}=\theta_{2}=\theta_{3}=\pi / 6$ and $\theta_{1}^{\prime}=\theta_{2}^{\prime}=\theta_{3}^{\prime}=-\pi / 2$, respectively. A structure containing six particles is shown in Fig. 2(b). We can see that the three colloidal particles at the corners are positioned in such a way that the rotational configurations are most likely to form. The magnetic configuration of the initial triangle (formed by the central particles) is not affected by the addition of the three extra macrospins. The angles between the magnetic moments of additional particles and their respective neighbors differ from $120^{\circ}$ [see the fifth and sixth particles on the right of Fig. 2(b)]. The magnetic moment of the top particle (sixth arrow) has an out-ofplane component, indicating that the stable configuration, in which all magnetic moments are in one plane, is not found. Figure 2(c) shows the situation after addition of the seventh particle. It can be seen that the hexagonal arrangement of the particles had to be broken in order to find an energetically favorable state. The top particle [see the sixth arrow in the right image of Fig. 2(c)] is shifted up and to the left compared to its initial position. On the other hand, the magnetic moment of this particle lies now in two dimensions, in contrast to the magnetic moment of the seventh particle, which is turned slightly out of plane.

A magnetically compensated structure appears when a cluster of 12 particles is formed [Fig. 2(d)]. The ordering of all particles in the system perfectly fits the triangular packing and all magnetic moments strictly lie in two dimensions. Second, the whole structure has three axes of symmetry [see Fig. 2(d) (right)]. All magnetic moments align with those axes, forming angles $\varphi=2 \pi / 3$ with respect to each other (the angles $\theta$ exhibits three different values: $\pi / 6,5 \pi / 6$, and $-\pi / 2$ ); thus only three preferred directions appear in this cluster. These facts distinguish the system of 12 particles from the systems imaged in Figs. 2(b) and 2(c), where not all particles are lined up with a fixed set of axes.

\section{Energy of the clusters}

The behavior of the clusters leads to the conclusion that magic clusters can be formed with three and twelve particles, 
while clusters containing six and seven (and all other numbers of particles between three and twelve, which are not discussed in detail here) form less stable clusters. This assumption is further supported by considering the pair interaction energies in these clusters. The mutual orientation of the magnetic moments in the three-particle cluster minimizes their pair interaction energies, which equal $E_{1}^{\text {rot }}=E_{2}^{\text {rot }}$ $=E_{3}^{\text {rot }}=-(1 / 2) a$ [Eq. (1)]. Therefore, this so called rotational triangle presents a ground state configuration with total energy $E^{\text {rot }}=-(3 / 2) a$, where the macrospins form the closed magnetic flux. The energy gained by the formation of the magic cluster stabilizes the particle arrangement. A similar magnetic behavior has been found in many two-dimensional triangular spin systems, where the ground state of the whole plaquette is reached by minimizing the energy of each separated fragment (the "rule of satisfied triangles") [32].

The orientation of the magnetic moments of the spheres that are added to form the six-particle cluster is determined by minimizing the energy of the whole cluster, including the added particle, simultaneously. Therefore, a configuration with a set of only local circulations cannot appear in the group of six particles. The formation of such a configuration would require energy for the rearrangement of the rotational triangle in the center of the cluster. Moreover, further considerations related to the chirality of magic clusters lead to the conclusion that a cluster consisting of six particles cannot be stabilized by only rotational configurations (see discussion of the 12-particle cluster below). Instead of this, three pair interactions characterized by the divergent orientations of the macrospins with $\theta_{1}=\theta_{2}=\theta_{3}=5 \pi / 6$ can be formed at the corners of the structure [see the arrows pointing to two of them in Fig. 2(b)]. Because each of the divergent pair interactions increases the total energy of the cluster by $E$ $=(7 / 4) a$, the ground state of the system is not reached by such a two-dimensional arrangement of the magnetic moments. To counterbalance it, the structure finds an alternative solution, where the final configuration contains one magnetic cap slightly tilted out of the plane, instead of one of the high-energy divergent contacts [see the upper particle in Fig. 2(b)]. The same issues define the behavior of the system after addition of the seventh colloidal particle to the cluster [Fig. 2(c)]. The system reaches its energetically favorable state by locally breaking the triangular packing. We can conclude that the system does not approach a state in which the magnetic interactions stabilize the spatial arrangement of the particles in the last two cases: the violation of the hexagonal symmetry and two-dimensional arrangement of the magnetic moments in the structure only brings the system to a state in which at least one particle (in this arrangement the topmost) fluctuates between two lattice sites. Such configurations are less stable than the magic configurations, meaning that they can be easily disturbed by the successive addition of colloidal particles to the cluster.

In the case of the 12-particle cluster, three macrospins in the center of the structure form a triangle with divergent pair interactions (a so-called divergent triangle) [Fig. 2(d)]. The energy can be evaluated from Eq. (1): $E^{\text {div }}=(21 / 4) a$. The surrounding three particles are needed to compensate this arrangement by pointing their magnetic moments to the center. This divergent arrangement consisting of six particles is

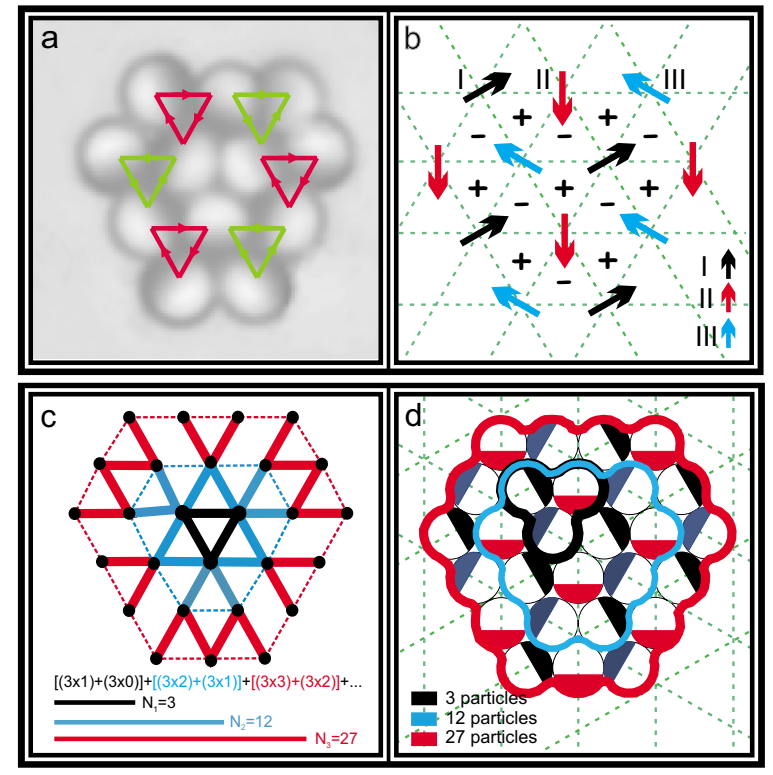

FIG. 3. (Color online) Images of magnetic clusters and schematic description of their properties. (a) is an image of the magic 12-particle cluster. The rotational centers of the structure are indicated by six triangles. The pink triangles display clockwise and green ones correspond to counterclockwise circulations in the structure. (b) Arrangement of the magnetic moments in the 12-particle cluster. Because the magnetic moments are aligned with the three axes of symmetry, three ferromagnetic sublattices of macrospins (four particles in each direction) can be considered in the cluster. Triangles with opposite chirality are designated + and -. (c) Schematic image illustrating the procedure of calculation of magic numbers. The black triangle corresponds to the initial nucleus; branching gives the next magic cluster with 12 particles (blue color), and so on. (d) displays a schematic view of the magic clusters. The boundaries of each cluster are marked with corresponding colors.

not stable by itself, as can be concluded from Fig. 2(b). Therefore, six additional particles are grouped around such a cell. Each of them follows the rules of arrangement set by the axes of symmetry. The presence of the additional six particles causes each macrospin to be a part of one of the six low-energy rotational triangles [each with energy $E^{\text {rot }}=-(3 / 2) a$ ], which form a ring [see Fig. 3(a)] around the high-energy divergent one $\left[E^{\mathrm{div}}=(21 / 4) a\right]$. It leads to the stabilization of the 12-particle cluster by minimizing the magnetic dipolar energy and to the appearance of an antiferromagnetic arrangement of macrospins on a triangular lattice. The last statement is explained and confirmed in Fig. 3.

\section{Frustrated antiferromagnet on triangular lattice}

Note that the clockwise and counterclockwise circulations of the magnetic flux in the rotational triangles are alternating and so fully compensated [Fig. 3(a)]. The orientations of all magnetic moments in the cluster are displayed schematically in Fig. 3(b). We can consider three groups of colloidal particles, which are distinguished by the directions of their magnetic moments. It is obvious that the same number of particles is aligned with each axis, forming three noncollinear ferromagnetic sublattices in the structure. These sublattices 
compensate each other, meaning that the magnetization of this 12-particle configuration equals zero. The magnetic moments of each sublattice are colored individually. Thus, this 12-particle cluster presents a stable state since the total energy is minimized by the unconventional antiferromagnetic order of the macrospins. Indeed, the specific character of this noncollinear magnetic arrangement in the cluster is caused by the geometry of the crystal lattice. As in two-dimensional spin systems, the ordering of the macroscopic magnetic moments on the triangular lattice is imposed by geometric frustration. It is well known that frustration-induced phases in spin systems on a triangular lattice have a chiral degree of freedom [12]; this means that the spin configuration of every triangle (a combination of any three magnetic moments interacting as NNs) on a lattice is degenerate (chiral degeneracy).

Naturally that chirality will also be peculiar to the ordering of the macrospins in the colloidal clusters. As shown in Fig. 3(b), the magnetic structure of the cluster is a set of triangles with opposite chirality, which are designated + (all rotational triangles with a divergent one in the center) and [isosceles triangles with energy $\left.E^{\text {isc }}=(3 / 4) a\right]$. The necessity of this alternation of + and - chiralities in the structure proves, in particular, that it is impossible to form a magnetic configuration with a set of only rotational triangles [see Fig. 2(b) discussed above].

\section{MAGIC CLUSTERS}

\section{A. Scheme of building of magic clusters}

During the experiment the formation of stable magic number clusters consisting of three and twelve colloidal particles was observed. Obviously, other magic clusters consisting of a larger number of particles should also exist. They can be obtained during the process of further growth of the existing structures. They must be distinguished by the same threefold symmetry and by the specific magnetic arrangement that minimizes the energy of the system. We propose a scheme for building magic clusters by taking into consideration the above-mentioned conditions. The procedure for obtaining a series of numbers which satisfy the criteria of stability is shown schematically in Fig. 3(c), where a branched structure, obtained from the central triangle in a stepwise manner, is presented. Each step forms the next family of branches. This construction helps to reconstitute the real process of cluster growth which took place in the experiment. The basic triangle initially has no branches; therefore the number of its knots corresponds to the magic number of the first order $\left(N_{1}=3\right)$. The second triangle, circumscribing the initial one, already belongs to the series, which describes the next magic number of second order. It has no branches but only knots at this stage. The magic number of second order is supplemented with the branches given by each knot of the basic triangle. Therefore, the second magic number equals 12 $\left(N_{2}=12\right)$. All following numbers can be obtained in the same way, and they equal $27,48,75, \ldots$. The general formula describing the stable magnetic cluster with magic number of $n$th order is given by

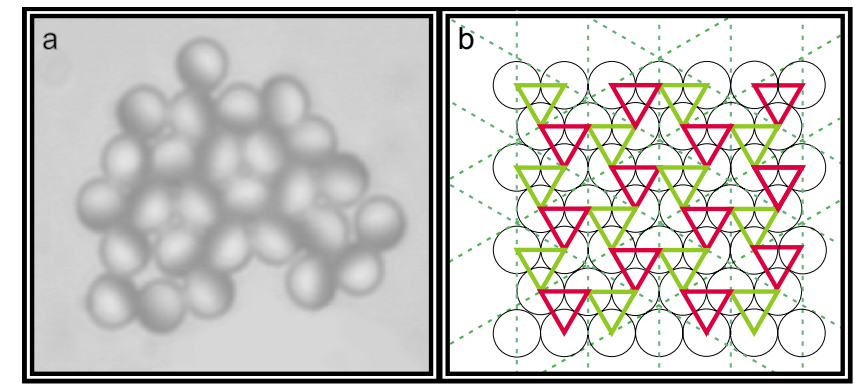

FIG. 4. (Color online) Magnetic arrangement of the infinite pattern. (a) displays a two-dimensional close-packed pattern with the triangular lattice formed by the magnetic colloidal particles. The magnetic arrangement of the space-filling pattern is identical with the ordering of the magnetic moments in a stable 12-particle cluster. (b) Schematic sketch of the antiferromagnetic space-filling pattern formed by the macrospins. The pink and green triangles correspond to rotational triangles with clockwise and counterclockwise circulations, respectively.

$$
N_{n}=3 / 2[(1+n) n+(n-1) n]=3 n^{2} .
$$

The lines in the sketch of Fig. 3(c) (continuous and dashed) represent the total number of nearest neighbor interactions in every magic cluster: three in the first one, 24 in the second, 63 in the third, and so on. Our assumption [Eq. (2)] is confirmed by Fig. 3(d), where a schematic view of magic clusters with corresponding numbers of particles 3,12 , and 27 is shown.

The stable magic clusters have properties which distinguish them from others. First of all they are characterized by the same symmetry group. All macrospins in magic clusters lie in two dimensions and their directions follow the rules of arrangement set by the axes of symmetry. In particular, the three-particle cluster represents a low-energy vortexlike configuration. The other 12- and 27-particle formations are stabilized by six and twelve rotational triangles, respectively, with alternating directions of the circulation of magnetic flux. Finally, the stable magnetic configurations in clusters are not affected by the addition of the successive particles as shown by Figs. 2(a)-2(d).

\section{B. Space-filling pattern}

The formation of clusters with perfect hexagonal symmetry denotes the possibility of the existence of a twodimensional space-filling pattern where the magnetic moments follow the same rules of arrangement as in the stable clusters. A small fragment of such a pattern was realized experimentally and is displayed in Fig. 4(a). The magnetic moments of all colloids lie strictly in the plane. However, the positions of many edge particles in the structure do not correspond to the ideal order provided by hexagonal packing. Moreover, the directions of the magnetic moments of these external particles do not coincide with the three preferred directions mentioned above. This misalignment is caused by the lower number of NNs in the particles at the edges of the structure. The lower number of pair interactions that the particles experience during random growth of the pattern leads 
to the appearance of defects in the hexagonal order and so to the deviations of the external macrospin orientations from the preferred axes. However, further growth of the pattern anneals the orientations and positions of the particles, gradually excluding structural defects. As illustrated in Fig. 4(b), the space-filling pattern also reaches its ground state through an antiferromagnetic arrangement. Nevertheless, the energy advantage, which leads to the equivalence of the magnetic configurations of the infinite system and of the magic number clusters, must yet be proven.

\section{CONCLUSIONS}

In conclusion, the formation of a colloidal cluster stabilized by magnetic interaction has been demonstrated. Stable, two-dimensional, close-packed magnetic clusters were obtained for a certain number of colloids in the cluster. It was shown that the magnetic structure of these "magic" clusters has zero flux and circulation of the magnetic moment. The ground state configurations that were observed experimentally match calculated structures, where the magnetic moments coincide with the centers of mass of the particles. This shows that the structures are robust against small changes in magnetic arrangement. Therefore the system can be used as a model system for a large variety of magnetically interacting particles.
Interacting macroscopic magnetic moments, as they are investigated here, are in many respects similar to the classical triangular antiferromagnet system, which is comprised of three ferromagnetic interpenetrating sublattices. Such an arrangement of macrospins in the system coincides with a ground state configuration that appears in an $X Y$-spin model on a triangular lattice. The most extensively studied triangular antiferromagnet is of $A B X_{3}$ type (for example, $\mathrm{YMnO}_{3}$ ). There is a large diversity of compounds with many possible combinations of the constituent atoms. The system of interacting magnetic colloidal particles studied here reconstitutes one of the triangular antiferromagnetic isolated layers of the magnetic ions $(B)$ in $A B X_{3}$ compounds. In addition to this, arrangements of the magnetic moments on lattices with diverse geometries (for example, square or rectangular), including those that have no experimental realization yet (e.g., Kagomé), can also be investigated with the technique presented here. This can be done by fixing the particles to certain positions by means of external potentials. Such potentials can be generated, for example, by structuring the substrate using anisotropic etching techniques or laser tweezers.

\section{ACKNOWLEDGMENT}

This work was supported by the International Research Training Group "Soft Condensed Matter."
[1] A. B. Schofield, P. N. Pusey, and P. Radcliffe, Phys. Rev. E 72, 031407 (2005).

[2] A. Yethiraj and A. van Blaaderen, Nature (London) 421, 513 (2003).

[3] E. V. Shevchenko, D. V. Talapin, N. A. Kotov, S. O'Brien, and Ch. Murray, Nature (London) 439, 55 (2006).

[4] K. Zahn, R. Lenke, and G. Maret, Phys. Rev. Lett. 82, 2721 (1999).

[5] U. Gasser, E. R. Weeks, A. Schofield, P. N. Pusey, and D. A. Weitz, Science 292, 258 (2001).

[6] P. Bartlett and A. I. Campbell, Phys. Rev. Lett. 95, 128302 (2005).

[7] Yi Gi-Ra et al., Adv. Mater. (Weinheim, Ger.) 16, 14 (2004).

[8] M. E. Leunissen et al., Nature (London) 437, 235 (2005).

[9] L. Néel, Ann. Phys. (Paris) 3, 137 (1948).

[10] G. Toulouse, Commun. Phys. (London) 2, 115 (1977).

[11] G. Toulouse, in Modern Trends in the Theory of Condensed Matter, edited by A. Pekalski and A. J. Przystawa (Springer, Berlin, 1980).

[12] H. Kawamura, J. Phys.: Condens. Matter 10, 4707 (1998).

[13] P. Fazekas and P. Anderson, Philos. Mag. 30, 423 (1974).

[14] I. Munawar and S. H. Curnoe, J. Phys.: Condens. Matter 18, 9575 (2006).

[15] V. P. Plakhty et al., Europhys. Lett. 48, 215 (1999).

[16] R. J. Birgeneau, M. A. Kastner, and A. Aharony, Z. Phys. B: Condens. Matter 71, 57 (1988).
[17] T. J. Sato, S. H. Lee, T. Katsufuji, M. Masaki, S. Park, J. R. D. Copley, and H. Takagi, Phys. Rev. B 68, 014432 (2003).

[18] S. Nakatsuji et al., Science 309, 1697 (2005).

[19] H. H. Hamdeh et al., J. Magn. Magn. Mater. 232, 175 (2001).

[20] J. Bogner et al., J. Magn. Magn. Mater. 226-230, 1319 (2001).

[21] N. Mohapatra, K. K. Iyer, S. Rayaprol, and E. V. Sampathkumaran, Phys. Rev. B 75, 214422 (2007).

[22] M. Grass, D. Fischer, M. Mathes, G. Ganteför, and P. Nielaba, Appl. Phys. Lett. 81, 3810 (2002).

[23] O. Echt, K. Sattler, and E. Recknagel, Phys. Rev. Lett. 47, 1121 (1981).

[24] F. Burmeister et al., Langmuir 13, 2983 (1997).

[25] L. Baraban, A. Erbe, and P. Leiderer, Eur. Phys. J. E 23, 129 (2007).

[26] M. Albrecht et al., Nat. Mater. 4, 203 (2005).

[27] T. Suzuki, Scr. Metall. Mater. 33, 1609 (1995).

[28] B. D. Terris, M. Albrecht, G. Hu, T. Thomson, and C. T. Rettner, IEEE Trans. Magn. 41, 2822 (2005).

[29] J. M. Shaw et al., J. Appl. Phys. 101, 023909 (2007).

[30] A. Ashkin and J. M. Dziedzic, Phys. Rev. Lett. 54, 1245 (1985).

[31] H. H. von Grünberg, L. Helden, P. Leiderer, and C. Bechinger, J. Chem. Phys. 114, 10094 (2001).

[32] E. F. Shender, V. B. Cherepanov, P. C. W. Holdsworth, and A. J. Berlinsky, Phys. Rev. Lett. 70, 3812 (1993). 